\title{
CARACTERÍSTICAS Y VISIBILIDAD DE LAS REVISTAS ESPAÑOLAS DE CIENCIAS DE LA SALUD EN BASES DE DATOS
}

\section{Features and visibility of Spanish health sciences journals in selected databases}

María-Francisca Abad-García, Aurora González-Teruel, Javier Argento y Josep-Manuel Rodríguez-Gairín

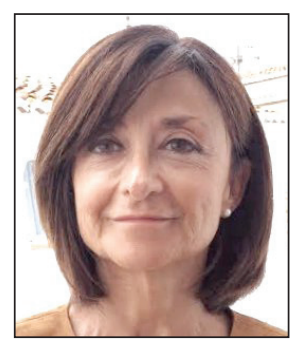

María-Francisca Abad-García es doctora en medicina, especialista en documentación médica y catedrática de biblioteconomía y documentación de la Universitat de València. Imparte docencia en medicina e información y documentación. Sus líneas de investigación preferentes versan sobre el proceso de comunicación científica, acceso abierto y evaluación de fuentes especialmente en ciencias de la salud. Durante más de diez años ha sido directora de la Biblioteca Virtual del Colegio de Médicos de Valencia y es uno de los integrantes del grupo de trabajo Accesoabierto.net. http://orcid.org/0000-0001-5611-4996

Universitat de València, Departamento de Historia de la Ciencia y Documentación Avda. Blasco Ibáñez, 15. 46010 Valencia, España abad@uv.es

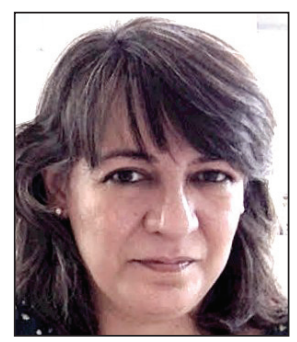

Aurora González-Teruel es profesora titular de biblioteconomía y documentación en el Departamento de Historia de la Ciencia y Documentación de la Universitat de València. Su investigación se centra en los fundamentos teóricos y metodológicos del comportamiento informacional así como en el proceso de comunicación científica y acceso abierto en el ámbito de ciencias de la salud. Participa en el proyecto de investigación sobre acceso abierto a la ciencia en España Accesoabierto.net. http://orcid.org/0000-0001-9304-2928

Universitat de València, Departamento de Historia de la Ciencia y Documentación Avda. Blasco Ibáñez, 15. 46010 Valencia, España agonzal@uv.es

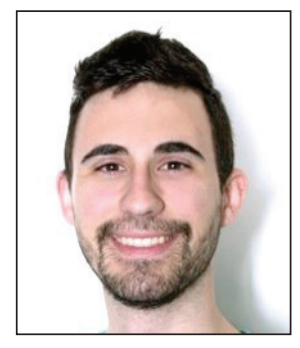

Javier Argento, graduado en información y documentación por la Universitat de València es coordinador del Col/lectiu de Documentalistes Universitaris. Ha sido becario de colaboración durante el curso académico 2014/2015 en el Departamento de Historia de la Ciencia y Documentación de la Universitat de València. Ha desempeñado trabajos en varias unidades de información centrándose en documentación y producción científica http://orcid.org/0000-0003-2918-0562

Universitat de València, Departamento de Historia de la ciencia y Documentación Avda. Blasco Ibáñez, 15. 46010 Valencia, España arvija@alumni.uv.es

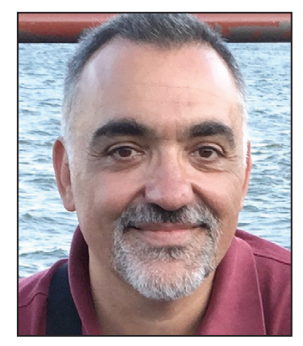

Josep-Manuel Rodríguez-Gairín es profesor titular de la Facultat de Biblioteconomia i Documentació de la Universitat de Barcelona, donde coordina las aulas de informática y asesora en aspectos tecnológicos. Ha llevado a cabo la infraestructura de proyectos como Revistas digitales de biblioteconomía y documentación (Temaria); revista BiD: Textos universitaris de biblioteconomia $i$ documentació; Matriz de información para la evaluación de revistas (Miar); Recursos en línia per elaborar treballs de recerca (Alehoop); Directorio de expertos en el tratamiento de la información (EXIT); International registry of authors-Links to identify scientists (IraLIS), etc. Es miembro de Ciepi, ThinkEPI y del consejo técnico del repositorio E-LIS. http://orcid.org/0000-0001-8375-7911

Universitat de Barcelona, Facultat de Biblioteconomia i Documentació Melcior de Palau, 140. 08014 Barcelona, España rodriguez.gairin@ub.edu 


\title{
Resumen
}

Se describen las características de 207 revistas españolas de ciencias de la salud activas en 2014 y su visibilidad en seis bases de datos biomédicas y multidisciplinares. Scopus e lbecs son las bases de datos que mayor número de revistas cubren, y Medline y WoS las que menos. La disciplina mejor representada es medicina, seguida de psicología, enfermería y farmacia. Se observa la práctica ausencia de revistas editadas exclusivamente en papel, la existencia de revistas publicadas en inglés, el aumento de versiones bilingües español-inglés y el acceso gratuito a los contenidos. El artículo incorpora el censo de revistas, que puede servir de referencia para los investigadores para seleccionar la revista dónde publicar, para los editores de revistas de ciencias de la salud, y para documentalistas que gestionan bases de datos o que asesoran a otros profesionales en las tareas mencionadas.

\section{Palabras clave}

Revistas españolas; Ciencias de la salud; Bases de datos; Visibilidad internacional; Acceso abierto; Ediciones bilingües; Idiomas.

\begin{abstract}
The main features of 207 Spanish health sciences journals active in 2014, and their visibility in six biomedical and multidisciplinary databases are described. Of these databases, Scopus and Ibecs provide the best coverage of these journals, and Medline and WoS the worst. The discipline best represented is medicine, followed by psychology, nursing and pharmacy. Among the observed characteristics, there were an absence of journals published exclusively on paper and the presence of journals published in English or bilingual Spanish-English and with open access. The article includes the journal list, which can be a reference tool for researchers selecting target journals for publication, for publishers in the health sciences areas and for information scientists for use in their database management tasks or in advising other professionals.
\end{abstract}

\section{Keywords}

Spanish journals; Health Sciences; Databases; International visibility; Open access; Bilingual editions; Languages.

Abad-García, María-Francisca; González-Teruel, Aurora; Argento, Javier; Rodríguez-Gairín, Josep-Manuel (2015). “Características y visibilidad de las revistas españolas de ciencias de la salud en bases de datos". El profesional de la información, v. 24, n. 5, pp. 537-550

http://dx.doi.org/10.3145/epi.2015.sep.04

\section{Introducción}

Las posibilidades actuales de internet para acceder a los artículos científicos mediante buscadores académicos, repositorios, redes sociales o desde las propias bases de datos (BDs) bibliográficas, han revolucionado el ecosistema tradicional de la comunicación científica. La emergencia de estas herramientas ha desdibujado el papel tradicional desempeñado por las revistas como punto de acceso y elemento integrador de los artículos científicos. Asimismo han generado nuevos hábitos de búsqueda y acceso a la información mermando el protagonismo de las BDs bibliográficas a favor de opciones más amigables de recuperación. (Giménez-Toledo; Rodríguez-Yunta, 2012). Aunque esta situación puede indicar que la revista ha pasado a un segundo plano, la ciencia moderna sigue atribuyéndole "la condición de ser el medio primordial de difusión de la ciencia, de constituir el registro oficial y público del conocimiento, de servir de certificación de la autoría y sobre todo, de asegurar que sus contenidos han sido sometidos a la consideración de otros científicos, a los que denominamos pares" (Ruiz-Pérez; Martín-Martín; Delgado-LópezCózar, 2015). Este proceso, aunque no es perfecto, es el mecanismo que mejor garantiza el control de calidad de lo publicado y contribuye decisivamente al prestigio de la revista como vehículo de comunicación.

A esto debe sumarse la apuesta que los sistemas de evaluación de la investigación hacen por este vehículo de comuni- cación, atribuyendo al artículo la calidad de la revista que lo publica. Este aspecto, en la mayoría de disciplinas, incluida la biomedicina, lo determina su inclusión en determinadas BDs y su posición en los índices de impacto. Estos criterios se fundamentan en el riguroso proceso que realizan algunas BDs para incluir sólo las revistas que cumplan determinados estándares, con la finalidad de captar únicamente aquellas en las que se publica "la corriente principal de la ciencia", como en el caso de WoS o para "captar sólo la investigación biomédica más importante y descartar el resto"(JiménezContreras, 2004) en el caso de Medline. Así la revistas "no sólo devienen en registro oficial, válido y público de la ciencia y constituyen el principal vehículo para difundir la información científica sino que se han convertido en una institución social que confiere prestigio y otorga recompensa a aquellos que contribuyen a su existencia" (Delgado-LópezCózar; Ruiz-Pérez; Jiménez-Contreras, 2007). Además, pese a que los artículos pueden ser localizados a través de búsquedas en la Web, las BDs aún son decisivas para asegurar la proyección y visibilidad de los contenidos de las revistas y siguen siendo "los intermediarios imprescindibles en el proceso de trasferencia de la información científica, y la búsqueda, recuperación, localización y obtención de los documentos pasa necesariamente por ellas" (Delgado-LópezCózar, Ruiz-Pérez; Jiménez-Contreras, 2007).

El número y características de las revistas existentes y su distribución en un campo científico determinado es indicativo de la especialización científica de un país (Sancho, 
2006). Los estudios realizados con el propósito de su identificación proporcionan resultados que trascienden su naturaleza censal constituyendo un reflejo de la conformación y consolidación de una disciplina científica en un momento determinado. Varios estudios han abordado estos objetivos en el campo de las ciencias de la salud (Vázquez-Valero et al., 2002; Ponce-Aura, 2004; Abad-García; González-Teruel; Martínez-Catalán, 2005; 2006; GimenoSieres, 2005; Llorente-Santacatalina, 2005; Osca et al., 2005, Sobrido-Prieto et al., 2005). Sus resultados aunque de vigencia limitada, son de una utilidad innegable para valorar las modificaciones de las revistas para adaptarse a los continuos cambios de su entorno, tanto científico, con la aparición de nuevas disciplinas y especialidades, como tecnológico y social, acaecidos sobre todo en las dos últimas décadas. Así, al desafío que planteó internet para la transformación de los formatos y soportes de las revistas, debe sumarse la emergencia del movimiento de acceso abierto (OA) que ha revolucionado el proceso de comunicación científica y que ha posibilitado el acceso gratuito a los contenidos de muchas revistas (Abad-García; González-Teruel; Martínez-Catalán, 2006). El OA ha supuesto también un cambio en los modelos de financiación de las revistas con la aparición de la posibilidad de que los autores paguen por la publicación de sus trabajos (Melero; Abad-García, 2008), así como la extensión de la reivindicación del acceso gratuito no sólo a los textos sino también a los datos que sustentan los resultados de las investigaciones (Melero; Hernández-San-Miguel, 2014).

\section{Es necesaria la realización de censos pe- riódicos de revistas para conocer su si- tuación actual y valorar los cambios que experimentan}

La necesidad de actualizar los datos sobre la situación actual de las revistas españolas de ciencias de la salud y conocer su evolución para adaptarse a los cambios antes mencionados ha conducido a la realización de este trabajo. Su objetivo es doble. Por una parte, elaborar un censo de las revistas españolas de ciencias de la salud activas en 2014 con visibilidad en las principales BDs nacionales e internacionales, tanto biomédicas como multidisciplinares y por otra analizar sus características.

Su finalidad es proporcionar información de referencia útil tanto para la toma de decisiones de los investigadores cuando seleccionen la revista donde publicar, de los editores cuando las valoren en el contexto de las existentes en su disciplina, y de los documentalistas que gestionan las BDs bibliográficas o asesoran a otros profesionales en las tareas mencionadas.

\section{Metodología}

Para la elaboración del censo se han incluido todas las revistas (excluyendo monografías y suplementos) cuyo país de edición sea España, de cualquier disciplina de ciencias de la salud, que durante 2014 hayan publicado al menos un número y que estén incluidas, al menos, en una de las siguientes BDs:

- Ibecs (Índice Bibliográfico Español de Ciencias de la Salud);

- Medes (Medicina en Español);

- Medline;

- Embase;

- Journal Citation Reports (ediciones Science y Social Science de 2013);

- Scopus.

La localización de las revistas se hizo en febrero de 2015 a partir de la lista de las publicaciones indizadas proporcionada por cada base de datos, comprobando de forma manual la pertenencia de cada revista al área de ciencias de la salud, el país de edición y si estaba o no activa. La particularidad del proceso en cada base de datos se describe a continuación y los resultados aparecen en la tabla 1.

Para las BDs Ibecs y Medes se utilizaron las listas de revistas indizadas presentes en sus webs. En el caso de Medline la búsqueda se realizó en el catálogo de la NLM mediante la ecuación: "currently indexed AND Spain [pl]"; y en el de Embase la selección se realizó mediante el filtrado, según país de edición, de los ficheros Excel que contienen la lista de las revistas indizadas.

http://www.ncbi.nlm.nih.gov/nlmcatalog

http://www.elsevier.com/solutions/embase/coverage

Las revistas de estas cuatro BDs se consideraron por defecto de ciencias de la salud y en todos los casos se comprobó su vigencia.

En el caso de la Web of Science, el estudio se ha centrado en las revistas incluidas en el Science Citation Index y Social Science Citation Index, y para su localización se utilizaron los Journal Citation Reports (JCR) (edición Science y Social Science) de 2013 que recogen las citas de los trabajos de los dos años anteriores. Por lo tanto, las incluidas en la base de datos con posterioridad al 2011 no aparecerán en las listas. La búsqueda en los JCR se realizó con la Suite Incites que permite su búsqueda conjunta según país de edición. De las 116 publicaciones resultantes se comprobó su temática de acuerdo con el título y política editorial de la revista y su actividad.

http://researchanalytics.thomsonreuters.com/incites

Para Scopus se consultó el archivo Excel de revistas indizadas, aplicando el filtro por país de edición, resultando 611 revistas españolas que se revisaron manualmente para seleccionar las de ciencias de las salud y de las que se comprobó posteriormente su actividad.

http://www.elsevier.com/online-tools/scopus/contentoverview

La lista final de revistas españolas de ciencias de la salud activas en el año 2014, fue de 216 , de las que se excluyeron nueve que, pese a estar editadas en España reflejan la actividad científica de Portugal (seis revistas) o Colombia (tres). La situación contraria, revistas que figuran como no españolas pero que sí podrían considerarse como tales por su responsabilidad en los contenidos no se ha investigado. El censo definitivo lo conforman 207 publicaciones. 
Tabla 1. Bases de datos fuente para la elaboración del censo de revistas españolas activas de ciencias de la salud. 2014

\begin{tabular}{|c|c|c|c|c|c|c|c|}
\hline Base de datos & Productor & $\begin{array}{l}\text { Cobertura } \\
\text { geográfica }\end{array}$ & $\begin{array}{l}\text { Cobertura } \\
\text { temporal }\end{array}$ & Cobertura temática & $\begin{array}{l}\text { Número } \\
\text { de revistas } \\
\text { indizadas }\end{array}$ & $\begin{array}{l}\text { Activas } \\
\text { españolas } \\
\text { ciencias } \\
\text { salud }\end{array}$ & $\begin{array}{c}\text { Aporte } \\
\text { absoluto al } \\
\text { censo } \\
\%\end{array}$ \\
\hline Ibecs & $\begin{array}{l}\text { Instituto de Salud } \\
\text { Carlos III / Bireme }\end{array}$ & España & $2000-$ & Ciencias de la salud & 218 & 155 & 74,9 \\
\hline Medes & Fundación Lilly & España & 2001- & $\begin{array}{l}\text { Revistas en español } \\
\text { de medicina, farmacia } \\
\text { enfermería }\end{array}$ & 87 & 77 & 37,2 \\
\hline Medline & $\begin{array}{l}\text { US National Library of } \\
\text { Medicine }\end{array}$ & Internacional & 1964- & Biomedicina & 5.623 & 62 & 30,0 \\
\hline Embase & Elsevier & Internacional & 1975- & Biomedicina & 5.940 & 91 & 44,0 \\
\hline WoS/JCR & Thomson Reuters & Internacional & $1900-$ & Interdisciplinar & 11.619 & 52 & 25,1 \\
\hline Scopus & Elsevier & Internacional & 1995- & Interdisciplinar & +21.000 & 160 & 77,3 \\
\hline
\end{tabular}

La información bibliográfica de cada una de las revistas del censo se completó con la base de datos del ISSN, con el catálogo colectivo Rebiun y con la información proporcionada por la revista en su sede web. Los aspectos analizados han sido:

- Cobertura de las BDs fuente: calculada para cada una de ellas como el porcentaje de las revistas incluidas por cada base de datos del total de revistas del censo. Los porcentajes serán los mismos que determinan el aporte de revistas de cada fuente al censo a excepción de los de Embase, ya que esta base de datos incorpora a su cobertura tanto las referencias de las publicaciones que ella misma indiza como las resultantes de la indización que realiza Medline de sus revistas, eliminando los duplicados entre ambas BDs.

- Número de BDs en las que están incluidas.

- Cobertura de revistas de cada base de datos según disciplina: Para la asignación de disciplinas se han tomado como referencia las disciplinas académicas actualmente vigentes relacionadas con las ciencias de la salud. Además a las revistas médicas se les ha asignado un área de especialización de acuerdo con criterios utilizados en otros estudios (Abad-García; González-Teruel; Martínez-Catalán, 2005) basados en el catálogo de especialidades MIR, en áreas de conocimiento propias del contexto académico (por ejemplo, anatomía, fisiología o historia de la medicina) o profesional (por ejemplo, administración y gestión hospitalaria, medicina militar). Además se ha tenido en cuenta una categoría multidisciplinar.

- Fecha de creación y antigüedad de la publicación: debido a la frecuencia de cambios en los títulos de las revistas el estudio de la antigüedad se ha realizado con relación a dos fechas: la fecha de aparición del título actual y la fecha en la que se creó la primera revista que da origen a la actual. Se ofrecen así mismo datos sobre la frecuencia en la que acontece el cambio de título en las publicaciones analizadas.

- Tipo editorial: se ha estudiado desde dos perspectivas, la del tipo de editor o responsable científico y la de la entidad responsable de su gestión y comercialización.

- Periodicidad y soporte: Para ambas variables inicialmente se ha utilizado como fuente la base de datos ISSN, si bien los datos se han comprobado consultando la web de la re- vista. Pese a ello cuando las revistas publican en formato online y en papel no siempre ha sido posible comprobar si la revista continúa o no con la publicación de su versión impresa, por lo que estos datos deberán leerse con cautela.

- Idioma: se ha estudiado desde la perspectiva del inglés analizando su uso como idioma en el título de la revistas y la presencia de ediciones bilingües español-inglés. Los datos se han obtenido de la revisión manual de todas las publicaciones.

- Tipo de acceso a los contenidos: se ha estudiado distinguiendo tres tipos de revistas: las de acceso gratuito, las que proporcionan acceso gratuito a su versión online pero mantienen la suscripción para su versión impresa y las de acceso por suscripción. En estas últimas se ha especificado la posibilidad de que los textos sean accesibles gratuitamente tras un período de embargo.

Además se han identificado las revistas que han adoptado un modelo de negocio basado en el pago de tasas de publicación. La información sobre el tipo de acceso y el modelo de negocio se ha obtenido de la consulta de la base de datos Dulcinea (Melero et al., 2009), que recoge los derechos de explotación y permisos para el auto-archivo de revistas científicas españolas. Cuando esta información no estaba disponible (en cinco ocasiones) se obtuvo de la propia revista.

\section{4 revistas están indexadas en todas las bases de datos estudiadas y constituyen la elite de las revistas españolas de cien- cias de la salud}

\section{Resultados}

\subsection{Aporte y cobertura de las BDs bibliográficas de referencia para el censo de revistas españolas de ciencias de la salud 2014}

El censo incluye 207 revistas españolas activas en 2014. Desde el punto de vista de su aporte (tabla 2), ninguna base de datos incluye en su lista de publicaciones el total de las identificadas. 
Tabla 2. Cobertura de las revistas españolas de ciencias de la salud por las bases de datos

\begin{tabular}{|l|c|c|c|}
\hline \multicolumn{1}{|c|}{ Base de datos } & Lista de revistas & Cobertura & $\%$ \\
\hline Scopus & 160 & 160 & 77,3 \\
\hline Ibecs & 155 & 155 & 74,9 \\
\hline Embase & 91 & 121 & 58,5 \\
\hline Medes & 77 & 77 & 37,2 \\
\hline Medline & 62 & 62 & 30,0 \\
\hline WoS & 52 & 52 & 25,1 \\
\hline
\end{tabular}

Las cifras de su cobertura (tabla 2) coinciden con las del aporte de sus listas a excepción de Embase, como ya se ha dicho: Esta base de datos cubre 121 revistas al sumar a sus propias publicaciones las de Medline eliminando el solapamiento entre ellas (32 revistas). Scopus, base de datos internacional y multidisciplinar, es la que mayor número de revistas de ciencias de la salud españolas recoge (77,3\%), seguida de la española especializada en esta área, lbecs y de la internacional de biomedicina, Embase. En el anexo 1 se muestra la lista de publicaciones con las bases de datos en las que están presentes.

\subsection{Bases de datos en las que figuran las revistas}

El 26\% de las revistas españolas de ciencias de la salud aparecen en una sola base de datos. Cuando esto sucede son sobre todo Ibecs y Scopus las que las incluyen. En la situación opuesta 24 revistas (11,6\%) están cubiertas por todas las BDs estudiadas.

Tabla 3. Revistas según el número de bases de datos en las que aparecen

\begin{tabular}{|c|c|c|c|c|c|c|c|c|}
\hline No BDs & Ibecs & Medes & Medline & Embase & WoS & Scopus & Total & $\%$ \\
\hline 1 & 30 & 0 & 0 & 6 & 0 & 18 & 54 & 26,1 \\
\hline 2 & 19 & 2 & 0 & 17 & 1 & 33 & 39 & 18,8 \\
\hline 3 & 31 & 10 & 2 & 22 & 4 & 33 & 36 & 17,4 \\
\hline 4 & 29 & 22 & 15 & 25 & 9 & 31 & 32 & 15,5 \\
\hline 5 & 22 & 19 & 21 & 27 & 14 & 21 & 22 & 10,6 \\
\hline 6 & 24 & 24 & 24 & 24 & 24 & 24 & 96 & 11,6 \\
\hline Total & 155 & 77 & 62 & 91 & 52 & 160 & 207 & \\
\hline
\end{tabular}

Tabla 4. Cobertura de las bases de datos de las disciplinas de ciencias de la salud

\begin{tabular}{|l|c|c|c|c|c|c|c|}
\hline & Ibecs & Medes & Medline & Embase & WoS & Scopus & Total \\
\hline $\begin{array}{l}\text { Medicina y sus espe- } \\
\text { cialidades }\end{array}$ & 110 & 66 & 51 & 97 & 37 & 106 & 140 \\
\hline Psicología & 15 & 0 & 1 & 3 & 9 & 19 & 24 \\
\hline Enfermería & 11 & 7 & 3 & 6 & 0 & 8 & 12 \\
\hline Farmacia & 5 & 2 & 2 & 9 & 2 & 11 & 11 \\
\hline Odontología & 7 & 0 & 1 & 1 & 1 & 6 & 9 \\
\hline Dietética y nutrición & 2 & 2 & 1 & 2 & 1 & 3 & 3 \\
\hline Logopedia & 1 & 0 & 0 & 0 & 0 & 2 & 2 \\
\hline Microbiología, mico- & 2 & 0 & 2 & 2 & 2 & 2 & 2 \\
\hline logía y parasitología & 0 & 0 & 0 & 0 & 0 & 1 & 1 \\
\hline Veterinaria & 1 & 0 & 0 & 0 & 0 & 1 & 1 \\
\hline Fisioterapia & 1 & 0 & 0 & 0 & 0 & 0 & 1 \\
\hline Optometría & & 0 & 1 & 1 & 0 & 1 & 1 \\
\hline Podología & & & & & & \\
\hline
\end{tabular}

Medline, WoS y Medes son las que acumulan el mayor número de revistas presentes en cuatro o más BDs $(86,8 \%$, $90,3 \%$ y $84,4 \%$ respectivamente), mientras que la proporción de las que cumplen esta condición en el caso de Scopus, Ibecs y Embase es mucho menor (47,5\%, 48,3\% y $62,8 \%$ respectivamente).

\subsection{Cobertura de las bases de datos según disciplinas científicas de las revistas de ciencias de la salud}

Las 207 revistas corresponden a 12 disciplinas de ciencias de la salud. La de mayor número de publicaciones es medicina $(67,6 \%)$, seguida de psicología (11,6\%), enfermería $(5,8 \%)$, farmacia $(5,3 \%)$ y odontología (4,3\%). El número para el resto de disciplinas varía entre 1 y 3 (tabla 4).

Entre las revistas de ciencias de la salud existe la tendencia de uso del inglés como estrategia para la internacionalización

El número de disciplinas cubiertas por las BDs es variable. Ibecs y Scopus, cubren 10 y 11 de las 12 disciplinas; Medline y Embase 8, WoS 6 y Medes sólo cuatro. La medicina es la disciplina, con diferencia, mejor cubierta por todas las BDs. La proporción de revistas médicas incluidas en Medes, Medline y Embase es similar (85,7\%; 82,3\% y $80 \%$ respectivamente) y superior a la que obtienen estas revistas en WoS, Ibecs, Scopus (71\% 70,5\% y 66\%) (gráfico 1). En cuanto a farmacia, son las bases de datos Embase e Ibecs las que mayor proporción de revistas integran en su cobertura, mientras que las de enfermería tienen un mayor peso específico en Medes e Ibecs. Por último cabe destacar que las revistas de psicología, dentro de las revistas de ciencia de la salud, ocupan un importante lugar en WoS, Scopus e Ibecs.

En cuanto al número de revistas, todas las BDs, menos Medes, incluyen una de las 24 revistas españolas de psicología, pero las que mejor cubren esta disciplina son Scopus con 19, Ibecs con 15 y WoS con 9.

En lo tocante a enfermería Ibecs, Scopus y Medes, con 11,9 y 7 , son las que mayor número de revistas indexan. No hay ninguna revista española de enfermería en la WoS. 
Todas las BDs incluyen al menos una revista española de farmacia. La mayor cobertura para esta disciplina la ofrecen Scopus, Embase e lbecs (11, 8 y 5 revistas respectivamente).

La revistas españolas de odontología están pobremente representadas en todas las BDs a excepción de Ibecs y Scopus y están ausentes de Medes. Sólo una está presente en WoS.

Por su parte, las publicaciones de dietética y nutrición y las de microbiología y parasitología figuran en prácticamente todas las BDs. Las revistas del resto de disciplinas están prácticamente ausentes de todas

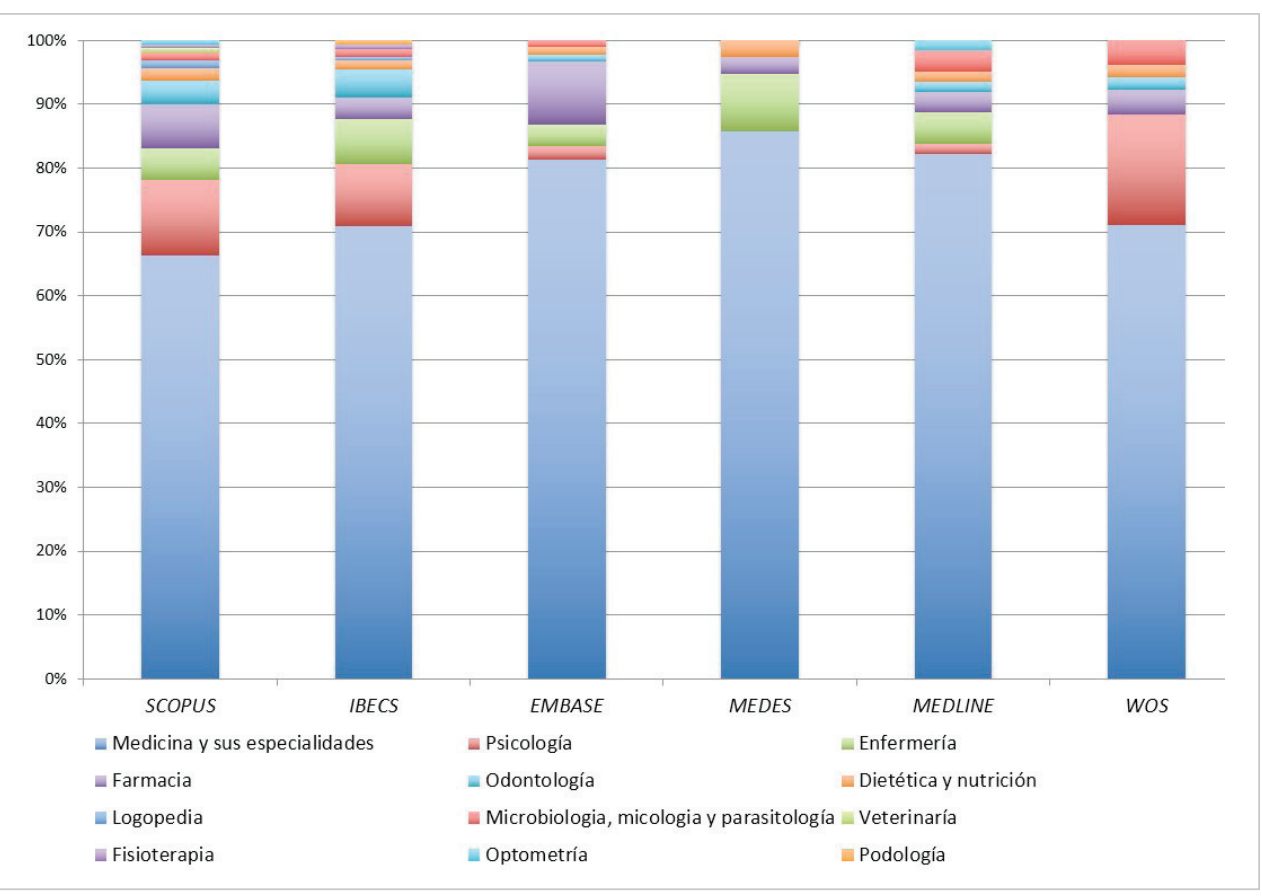

Gráfico 1. Distribución porcentual de las disciplinas en las bases de datos estudiadas

Tabla 5. Especialización de las revistas españolas de medicina

\begin{tabular}{|c|c|c|}
\hline Especialidades & No de revistas & $\%$ \\
\hline Pediatría & 9 & 6,2 \\
\hline Psiquiatría & 9 & 6,2 \\
\hline Medicina interna & 6 & 4,1 \\
\hline Medicina preventiva y salud pública & 5 & 3,4 \\
\hline Neurología & 5 & 3,4 \\
\hline Alergología e inmunología & 4 & 2,8 \\
\hline Cirugía general y del aparato digestivo & 4 & 2,8 \\
\hline Dermatología y venereología & 4 & 2,8 \\
\hline Drogodependencias & 4 & 2,8 \\
\hline Medicina del deporte & 4 & 2,8 \\
\hline Medicina familiar y comunitaria & 4 & 2,8 \\
\hline Medicina legal y forense & 4 & 2,8 \\
\hline Medicinas alternativas & 4 & 2,8 \\
\hline Multidisciplinar & 4 & 2,8 \\
\hline Obstetricia y ginecología & 4 & 2,8 \\
\hline Urología & 4 & 2,8 \\
\hline Cirugía ortopédica y traumatología & 3 & 2,1 \\
\hline Enfermedades infecciosas & 3 & 2,1 \\
\hline Historia de la medicina & 3 & 2,1 \\
\hline Medicina del trabajo & 3 & 2,1 \\
\hline Neumología & 3 & 2,1 \\
\hline Oncología & 3 & 2,1 \\
\hline Reumatología & 3 & 2,1 \\
\hline Administración y gestión sanitaria & 2 & 1,4 \\
\hline Anatomía & 2 & 1,4 \\
\hline Anestesiología y reanimación & 2 & 1,4 \\
\hline Angiología y cirugía vascular & 2 & 1,4 \\
\hline Aparato digestivo & 2 & 1,4 \\
\hline
\end{tabular}

\begin{tabular}{|c|c|c|}
\hline Especialidades & No de revistas & $\%$ \\
\hline Cardiología & 2 & 1,4 \\
\hline Endocrinología y nutrición & 2 & 1,4 \\
\hline Geriatría & 2 & 1,4 \\
\hline Medicina del dolor & 2 & 1,4 \\
\hline Nefrología & 2 & 1,4 \\
\hline Oftalmología & 2 & 1,4 \\
\hline Patología e histología & 2 & 1,4 \\
\hline Radiodiagnóstico & 2 & 1,4 \\
\hline Cirugía cardiovascular & 1 & 0,7 \\
\hline Cirugía pediátrica & 1 & 0,7 \\
\hline Cirugía plástica, estética y reparadora & 1 & 0,7 \\
\hline Cirugía torácica & 1 & 0,7 \\
\hline Educación médica & 1 & 0,7 \\
\hline Fisiología y bioquímica & 1 & 0,7 \\
\hline Investigación & 1 & 0,7 \\
\hline Laboratorio clínico & 1 & 0,7 \\
\hline Medicina de emergencias & 1 & 0,7 \\
\hline Medicina del sueño & 1 & 0,7 \\
\hline Medicina intensiva & 1 & 0,7 \\
\hline Medicina militar & 1 & 0,7 \\
\hline Medicina nuclear & 1 & 0,7 \\
\hline Medicina penitenciaria & 1 & 0,7 \\
\hline Microbiología y parasitología & 1 & 0,7 \\
\hline Neurocirugía & 1 & 0,7 \\
\hline Otorrinolaringología & 1 & 0,7 \\
\hline Rehabilitación & 1 & 0,7 \\
\hline Toxicología & 1 & 0,7 \\
\hline Traducción médica & 1 & 0,7 \\
\hline Total & 145 & 100 \\
\hline
\end{tabular}


Tabla 6. Distribución de la antigüedad de las revistas según año del título inicial y del título actual

\begin{tabular}{|l|c|c|c|c|}
\hline Períodos & $\begin{array}{c}\text { Título } \\
\text { inicial }\end{array}$ & $\%$ & $\begin{array}{c}\text { Título } \\
\text { actual }\end{array}$ & $\%$ \\
\hline $1900-1949$ & 18 & 8,7 & 10 & 4,8 \\
\hline $1950-1969$ & 26 & 12,6 & 10 & 4,8 \\
\hline $1970-1979$ & 21 & 10,1 & 15 & 7,2 \\
\hline $1980-1989$ & 51 & 24,6 & 45 & 21,7 \\
\hline $1990-1999$ & 51 & 24,6 & 64 & 30,9 \\
\hline $2000-2009$ & 35 & 16,9 & 51 & 24,6 \\
\hline $2010-$ & 5 & 2,4 & 9 & 4,3 \\
\hline Total & 207 & 100,0 & 207 & 100,0 \\
\hline
\end{tabular}

las BDs, excepto de Ibecs y Scopus, cuyo perfil se revela más multidisciplinar que el resto.

\subsection{Especialidad de las revistas médicas}

Las 140 revistas médicas se han clasificado en 56 áreas de especialización. Cinco se han clasificado en más de un área (tabla 4). El rango de áreas es muy amplio y abarca desde disciplinas y especialidades tradicionales hasta superespecialidades como medicina del dolor o del sueño, o incluso áreas no médicas pero relacionadas con ella como la de traducción médica. En la categoría multidisciplinar se han clasificado revistas que así lo especifican en su política editorial como, por ejemplo, las dos sobre el síndrome de Down.

Treinta y cinco especialidades tienen más de 1 revista, siendo pediatría y psiquiatría las de mayor número, con 9 cada una, seguidas de medicina general e interna, medicina preventiva y salud pública y neurología, con 6 y 5 respectivamente.

\subsection{Antigüedad de las revistas españolas de ciencias de la salud}

La tabla 6 ofrece la distribución de las revistas de acuerdo con dos fechas, la del origen de la revista y la del título actual. Así, de acuerdo con la fecha inicial de creación se observa que 19 publicaciones tienen una trayectoria que comenzó durante la primera mitad del siglo $X X$, el origen del $50 \%$ de las mismas se sitúa en las décadas 80 y 90 y se han creado 40 nuevas desde el principio del siglo XXI, de las que 5 corresponden al último quinquenio.

Tabla 7. Distribución de las revistas según tipo y función editorial

\begin{tabular}{|l|c|c|c|c|}
\hline \multicolumn{1}{|c|}{ Tipo de editorial } & $\begin{array}{c}\text { Responsable } \\
\text { científico de los } \\
\text { contenidos }\end{array}$ & $\%$ & $\begin{array}{c}\text { Responsable de } \\
\text { distribución y co- } \\
\text { mercialización }\end{array}$ & $\%$ \\
\hline Sociedades científicas & 121 & 58,5 & 39 & 18,8 \\
\hline Editoriales comerciales & 30 & 14,5 & 125 & 60,4 \\
\hline $\begin{array}{l}\text { Universidades y centros de } \\
\text { investigación }\end{array}$ & 20 & 9,7 & 19 & 9,2 \\
\hline $\begin{array}{l}\text { Academias ciencias + colegios } \\
\text { profesionales }\end{array}$ & 15 & 7,2 & 9 & 3,3 \\
\hline Fundaciones & 10 & 4,8 & 8 & 1,9 \\
\hline Estamentos gubernamentales & 4 & 1,9 & 4 & 1,4 \\
\hline Otros & 7 & 3,4 & 3 & 100,0 \\
\hline Total & 207 & 100,0 & 207 & \\
\hline
\end{tabular}

La distribución según la fecha del título actual evidencia el efecto que los cambios de título tiene en la antigüedad de las revistas y que han afectado a 68 de las 207 (32,9\%). De ellas 46 lo han cambiado una vez y 22 lo han hecho dos. Un ejemplo es el de Archivos de oftalmología hispano-americanos, la más antigua identificada que se creó en 1901, en 1942 pasó a llamarse Archivos de la Sociedad Oftalmológica Hispano-Americana y en 1971 adoptó su título actual de Archivos de la Sociedad Española de Oftalmología.

\subsection{Tipo editorial}

Se han agrupado en cinco categorías, considerando para cada una sus funciones, la responsabilidad del contenido científico de la revista y la de su gestión y comercialización (tabla 7).

Los editores científicos son con mayor frecuencia las sociedades científicas $(58,5 \%)$ que junto con las universidades y centros de investigación, las academias y colegios profesionales y las fundaciones científicas suman el $80,2 \%$ de las revistas. Las editoriales comerciales asumen este papel sólo en 29 revistas.

Scopus $(77,3 \%)$ e Ibecs $(74,9 \%)$ son las BDs que cubren el mayor número de revistas

Las tareas de comercialización y distribución de las revistas recaen fundamentalmente en entidades comerciales $(60,4 \%)$ tanto para las revistas que éstas impulsan como sobre todo, para las creadas por las sociedades científicas. Cuando las publicaciones pertenecen a universidades y centros de investigación, colegios profesionales, fundaciones o centros gubernamentales éstas entidades suelen cumplir ambas funciones.

Se han identificado 32 editoriales comerciales. Elsevier lidera la edición de revistas españolas de ciencias de la salud, con 74 revistas que representan el $59,2 \%$ de las 125 revistas de esa categoría (tabla 8 ) y el $35,7 \%$ del censo total. Otras 7 revistas están editadas por potentes grupos editoriales internacionales como Springer, Thomson Reuters, Cambridge University Press o Taylor \& Francis. El resto de las editoriales comerciales publica entre una y cuatro revistas.

\subsection{Periodicidad en la publica- ción}

El $85 \%$ de las revistas de ciencias de la salud españolas se publican en intervalos superiores a un mes y casi la mitad de ellas se publican con una periodicidad trimestral $(33,8 \%)$ o cuatrimestral $(15,9 \%)$.

\subsection{Tipo de soporte}

En cuanto al tipo de soporte, la mayoría, 179 (86,5\%), se editan en papel y en formato electrónico. Las revistas exclusivamente electrónicas son 27 (13\%). Con edición úni- 
Tabla 8. Editoriales comerciales de las revistas españolas activas de ciencias de la salud

\begin{tabular}{|l|c|c|}
\hline Editoriales comerciales & $\begin{array}{c}\text { No de } \\
\text { revistas }\end{array}$ & $\%$ \\
\hline Elsevier & 74 & 59,2 \\
\hline Grupo Arán & 4 & 3,2 \\
\hline Publicaciones Peyamner & 4 & 3,2 \\
\hline Ediciones Mayo & 3 & 2,4 \\
\hline Ergón & 3 & 2,4 \\
\hline Springer & 3 & 2,4 \\
\hline Viguera Editores SLU & 3 & 2,4 \\
\hline Arké & 2 & 1,6 \\
\hline Avances SL & 2 & 1,6 \\
\hline Ediciones ROL & 2 & 1,6 \\
\hline iMedPub & 2 & 1,6 \\
\hline Medicina Oral S.L. & 2 & 1,6 \\
\hline Thomson Reuters & 2 & 1,6 \\
\hline Aula Médica & 1 & 0,8 \\
\hline Benito Menni, Complex Assistencial en Salut Mental & 1 & 0,8 \\
\hline Cambridge University Press & 1 & 0,8 \\
\hline & & \\
\hline
\end{tabular}

\begin{tabular}{|l|c|c|}
\hline Editoriales comerciales & $\begin{array}{c}\text { No de } \\
\text { revistas }\end{array}$ & $\%$ \\
\hline Difusión Avances de Enfermería & 1 & 0,8 \\
\hline Edimsa - Editores Médicos SA & 1 & 0,8 \\
\hline Editorial Glosa & 1 & 0,8 \\
\hline Editorial Médica & 1 & 0,8 \\
\hline Centro de Investigaciones y Publicaciones Farmacéuticas & 1 & 0,8 \\
\hline Esmon Publicidad SA & 1 & 0,8 \\
\hline Grupo Editorial Nefrología & 1 & 0,8 \\
\hline Horsori Editorial SL & 1 & 0,8 \\
\hline Ibáñez \& Plaza Editorial & 1 & 0,8 \\
\hline Idemm Farma SL & 1 & 0,8 \\
\hline Iniestares SAU & 1 & 0,8 \\
\hline Lua 3.O. Ediciones & 1 & 0,8 \\
\hline Puntex & 1 & 0,8 \\
\hline Rasgo Editorial S.L. & 1 & 0,8 \\
\hline Talleres Editoriales Cometa, S.A. & 1 & 0,8 \\
\hline Taylor \& Francis & 125 & 0,8 \\
\hline Total & 100 \\
\hline
\end{tabular}

camente en papel sólo hay una, la Revista Fontilles, creada en 1932.

\subsection{Idioma de publicación}

De las 207 revistas, 26 (12,5\%) tienen el título en inglés. De ellas 19 publican sólo en este idioma, 2 tienen una versión bilingüe inglés-español, 3 admiten trabajos en ambos idiomas y 2 sólo artículos en español. De las 181 con título en español 35 tienen una versión bilingüe español-inglés.

\subsection{Tipo de acceso a los contenidos}

$92(44,4 \%)$ revistas son de acceso gratuito (sólo en una ocasión la gratuidad del acceso conlleva para los autores el pago de tasas de publicación), 24 (11,6\%) son de acceso gratuito en la versión online y de suscripción en la versión en papel y 91 (44\%) son revistas por suscripción.

De las revistas por suscripción, 66 no permiten el acceso a ningún documento, 20 permiten el acceso tras un período variable de embargo y 5 funcionan con un modelo híbrido

Tabla 9. Periodicidad de las revistas españolas de ciencias de la salud

\begin{tabular}{|l|c|c|}
\hline \multicolumn{1}{|c|}{ Periodicidad } & No de revistas & $\%$ \\
\hline Semanal & 2 & 1,0 \\
\hline Quincenal & 1 & 0,5 \\
\hline Mensual & 29 & 14,0 \\
\hline Bimestral & 39 & 18,8 \\
\hline Trimestral & 70 & 33,8 \\
\hline Cuatrimestral & 33 & 15,9 \\
\hline Semi-anual & 28 & 13,5 \\
\hline Anual & 3 & 1,4 \\
\hline Irregular & 2 & 1,0 \\
\hline TOTAL & $\mathbf{2 0 7}$ & 100,0 \\
\hline
\end{tabular}

que permite el acceso a los textos para los que los autores han pagado tasas de publicación para liberar los artículos.

Los períodos de embargo de las 20 revistas son variables, siendo en 9 de entre 3 y 6 meses, en 8 de un año y en 3 de tres años desde la fecha de publicación del trabajo.

En conjunto la posibilidad de acceso gratuito a los textos lo proporcionan 116 revistas (56\%), cifra que asciende a 136 $(65,7 \%)$ cuando se suma el acceso tras un período de embargo.

El acceso gratuito a los artículos publicados en las revistas de ciencias de la salud españolas es posible en el $56 \%$ de las revistas que están en estas bases de datos

\section{Discusión}

Se han analizado las características y visibilidad de 207 revistas de ciencias de la salud españolas que representan un $34,9 \%$ de las 471 de ciencias de la salud que estaban activas en el año 2000 (Vázquez-Valero et al., 2002).

Scopus $(77,3 \%)$ e Ibecs $(74,9 \%)$ son las BDs que cubren el mayor número, coincidiendo con el propósito de exhaustividad que ambas declaran:

- Ibecs para las revistas españolas de ciencias de la salud (Bojo-Canales; Jaén-Casquero, 2003);

- Scopus en su objetivo de ser "una herramienta de navegación internacional de ciencia, tecnología, medicina y ciencias sociales, con una distribución geográfica y equitativa de los títulos" (Rozenblum et al., 2015). 
Que Scopus, una BD internacional y multidisciplinar incluya más revistas españolas que cualquiera de las dos bases de datos españolas analizadas no deja de sorprender y plantea la necesidad de indagar si éste es un hecho singular que afecta sólo a las revistas españolas de ciencias de la salud o si también ocurre con las publicaciones españolas de otras disciplinas. Scopus añade a la visibilidad internacional de estas revistas la capacidad de proporcionar las citas que reciben sus trabajos y de obtener de indicadores bibliométricos útiles para la justificación del impacto de la producción investigadora, en una firme competencia por el papel que hasta este momento ha jugado WoS.

Por su parte Ibecs, patrocinada por la Biblioteca Virtual de Salud Española, permite una difusión tanto en el ámbito nacional como entre los países de habla hispana al formar parte de la iniciativa Bireme (OMS/OPS), añadiendo como ventaja la gratuidad en el acceso (Bojo-Canales; Jaén-Casquero, 2003).

En la situación opuesta están Medline y WoS con 62 (30\%) y $52(25 \%)$ revistas, las de mayor prestigio y las más codiciadas por las publicaciones, por su papel tanto en la visibilidad internacional (Medline es la base de datos más utilizada por los profesionales de la biomedicina) como por la aplicación de WoS en los procesos de evaluación. Pese a que Medline incorpora más revistas que WoS el ritmo de incorporación es mucho más lento. De hecho, las 62 publicaciones cubiertas por Medline son sólo 20 más de las 40 indizadas en el año 2000 (Ponce-Aura, 2004), lo que representa un incremento medio anual de 1,4 revistas, mientras que la presencia de estas revistas se ha duplicado en WoS en la última década, a tenor de las 25 de ciencias de la salud que identifica la consulta de los JCR de 2003.

\section{Las revistas exclusivamente en papel no están presentes en las bases de datos de referencia y por lo tanto son invisibles}

La BD española Medes cubre un porcentaje de revistas similar a las anteriores (37,2\%), lo que se justifica porque su cobertura está restringida a las publicaciones españolas en español sólo de algunas disciplinas.

http://www.fundacionlilly.com/es/actividades/medes-medicinaen-espaniol/base-de-datos-medes.asp

Por su parte Embase, con un 58\% de las revistas, consigue ser la base de datos biomédica internacional más exhaustiva al aunar en una sola plataforma las publicaciones que ella misma indiza con las de Medline. Cabe mencionar también que aunque Embase y Scopus tengan el mismo productor (Elsevier), los datos de cobertura de revistas españolas de ciencias de la salud son dispares, lo que revela el uso de distintos criterios de selección.

Desde la perspectiva de las revistas destaca que un $26 \%$ aparece en una sola base de datos y que cuando esto ocurre lo hacen en Scopus o Ibecs. Por el contrario sólo 24 (11,6\%) están incluidas en todas las BDs estudiadas y representan la élite de las revistas de ciencias de la salud españolas.
Se han identificado revistas de 12 disciplinas, correspondiendo a la medicina el mayor número con 140. Esta cifra representa el 38,9\% de las 360 revistas médicas activas en 2004 (Abad-García; González-Teruel; Martínez-Catalán, 2005). Medes, Medline y Embase son las BDs con mayor proporción de revistas médicas. Sólo 51 revistas (36\%) están incluidas en Medline y 37 (26,4\%) en WoS. Las 24 revistas presentes en todas las BDs (anexo 1) son todas de medicina.

La gran variedad de temas revela un alto grado de especialización reflejo, en muchas ocasiones, de la práctica profesional actual. Es el caso, por ejemplo, de las revistas de medicina del dolor o del sueño. También hay revistas cuya base conceptual no es médica pero sí lo es su ámbito de aplicación como las revistas de traducción y de educación médica. La existencia de varias publicaciones para una misma especialidad, es un hecho ya constatado en estudios anteriores (Abad-García; González-Teruel; Martínez-Catalán, 2005) que puede crear situaciones de competencia en la atracción de originales y poner en riesgo su posicionamiento en las BDs de mayor relevancia, salvo en el caso de revistas con contenidos claramente diferenciados y con una importante población de investigadores potenciales

Las 24 revistas de psicología contabilizan el 23\% de las 102 publicaciones de psicología y psiquiatría identificadas en el estudio de Osca et al. (2005) usando 15 fuentes de información. Estas revistas están presentes sobre todo en Scopus y WoS.

En lo tocante a enfermería se han hallado 12 revistas, un escaso número si se consideran las 116 que en 2005 Sobrido-Prieto et al. encontraron utilizando 7 bases de datos, de ellas 5 nacionales (Cuiden, Cuidatge, BDIE, Enfispo e IME) y 2 internacionales (Cinahl, y Medline). Destaca que al igual que en el estudio mencionado sólo 3 revistas están indexadas en Medline, por lo que la visibilidad de estas publicaciones en esta BD no ha mejorado, y que ninguna está presente en WoS. La visibilidad internacional de estas revistas se la presta Scopus, que incluye 7 de ellas.

La revistas españolas de odontología están pobremente representadas en todas las BDs a excepción de Ibecs y Scopus y están ausentes de Medes. En Medline, Embase y WoS sólo hay una.

Farmacia, pese a ser una disciplina con gran tradición en España y con revistas iniciadas en el siglo XIX (De-Jaime-Lovén; Ruiz-Ovejero, 1998) está representada en las BDs estudiadas con 11 revistas, que figuran sobre todo en Scopus y Embase. Sólo dos están incluidas en Medline y WoS.

Las revistas de dietética y nutrición y las de microbiología y parasitología son pocas pero están incluidas en casi todas las BDs y las del resto de disciplinas están prácticamente ausentes de todas ellas, excepto de Ibecs y Scopus.

Algunas características de las revistas estudiadas muestran poca variación con lo descrito por Vázquez-Valero et al. (2002). Esto es el caso de la periodicidad o las fecha de creación, debido a que una gran parte de ellas está presente en ambos censos. Cabe reseñar no obstante, que se han creado 40 revistas desde el comienzo del siglo XXI. Tampoco ha experimentado variaciones el predominio que tienen como editor científico las sociedades científicas, universidades y 
colegios profesionales y el de las editoriales comerciales para su difusión y comercialización.

Otras características evidencian sin embargo su evolución, destacando que las revistas exclusivamente en papel no se encuentran por las BDs de referencia y por lo tanto son invisibles. Pese a eso el abandono del papel no es un hecho ya que sólo un $14,5 \%$ se editan en soporte exclusivamente electrónico, siendo habitual la convivencia de los dos soportes. Hay que destacar la dificultad de obtener datos fiables sobre este hecho pues las revistas no siempre informan del abandono del papel a las fuentes de referencia como el ISSN y raramente anuncian este hecho en su web.

Otro hecho reseñable es la progresiva adopción del inglés por las revistas españolas de ciencias de la salud, destacando su uso como lengua vehicular en 19 revistas y la adopción de versiones bilingües en 37 . El uso del inglés es una de las estrategias de las revistas para aumentar su internacionalización, incrementando el número de potenciales lectores y la posibilidad de recibir artículos de autores extranjeros (Cremades-Pallas et al., 2013). La versión bilingüe permite conservar aquellos lectores en español habituados al uso de esta lengua (González-de-Dios et al., 2011) y su difusión entre los países hispanohablantes. No obstante esa estrategia supone dificultades como el desfase entre ambas versiones resultado de la traducción los textos que puede ser de entre 30 y 90 días (Fecyt, 2013) que repercute en una pérdida de actualidad de la versión inglesa. Otra más evidente es el coste editorial adicional que representa la traducción, por lo que sólo lo podrán adoptar las revistas con un sistema de financiación saneado.

Finalmente otro aspecto reseñable es la posibilidad de acceder gratuitamente a los textos, y los modelos de negocio que adoptan las revistas para su consecución. El acceso gratuito a los artículos publicados en las revistas de ciencias de la salud españolas es posible en el $56 \%$ de las revistas presentes en estas BDs. Este dato corrobora la tendencia ya evidenciada en 2004 para las revistas médicas españolas en las que el 63\% disponían de acceso electrónico (Abad-García; González-Teruel; Martínez-Catalán, 2005). La gratuidad en el acceso es tanto para lectores como para autores, ya que sólo en un caso el acceso es consecuencia de un modelo de pago por autor. Finalmente, de entre las revistas por suscripción, sólo 5 son de modelo híbrido (acceso por suscripción y acceso gratuito a artículos cuyos autores hayan pagado las tasas de publicación).

\section{Agradecimientos}

Esta investigación se ha realizado en el marco del proyecto El acceso abierto a la producción científica (open access) en España: análisis del grado de implantación y de la sostenibilidad de un nuevo modelo de comunicación científica, financiado por el Gobierno de España. Ministerio de Ciencia e Innovación. Plan Nacional de I+D (CSO2011-29503-C02-02).

\section{Bibliografía}

Abad-García, María-Francisca; González-Teruel, Aurora; Martínez-Catalán, Celeste (2005). "Características de las revistas médicas españolas. 2004". El profesional de la información, v. 14, n. 5, pp. 380-390. http://www.elprofesionaldelainformacion.com/contenidos/2005/ septiembre/9.pdf

Abad-García, María-Francisca; González-Teruel, Aurora; Martínez-Catalán, Celeste (2006). "Acceso abierto y revistas médicas españolas". Medicina clínica, v. 127, n. 12, pp. 456-464. http://digital.csic.es/handle/10261/4113 http://dx.doi.org/10.1157/13093056

Bojo-Canales, Cristina; Jaén-Casquero, Belén (2003). "Principales bases de datos en la Biblioteca Virtual en Salud - España". En: VI Congreso nacional de informática de la salud. Madrid, 2-4 de abril de 2003, pp. 81-86.

http://www.conganat.org/SEIS/inforsalud03/INFORSALUD2003_ bojoc.pdf

Cremades-Pallas, Rebeca; Burbano-Santos, Pablo; Valcárcel-de-la-Iglesia, María-Ángel; Burillo-Putze, Guillermo; Martín-Sánchez, Francisco-Javier; Miró, Òscar (2013). “Impacto de la inclusión de artículos escritos en revistas biomédicas en ediciones multilingües". Anales del sistema sanitario de Navarra, v. 36, n. 3, pp. 467-470.

http://recyt.fecyt.es/index.php/ASSN/article/view/22115

De-Jaime-Lorén, José-María; Ruiz-Ovejero, Álvaro (1998). El mensual farmacéutico (1842-1843): primera revista española de farmacia, obra del briviescano Carlos Mallaina. Valencia: José María De Jaime Lorén.

http://hdl.handle.net/10637/7238

Delgado-López-Cózar, Emilio; Ruiz-Pérez, Rafael; JiménezContreras, Evaristo (2007). La edición de revistas científicas. Directrices, criterios y modelos de evaluación, Madrid: Fundación Española para la Ciencia y la Tecnología, ISBN 848198.

http://www.fecyt.es/es/system/files/publications/ attachments/2014/11/la_edicion_de_revistas_cientificas._ directrices_criterios_y_modelos_de_evaluacion.pdf

Fecyt (2013). Manual de buenas prácticas en edición de revistas científicas. Madrid: Fundación Española para la Ciencia y la Tecnología, Fecyt. NIPO: 720120698

http://www.fecyt.es/es/system/files/publications/ attachments/2014/11/manual-de-buenas-practicas-en-laedicion-de-revistas-cientificas.pdf

Giménez-Toledo, Elea; Rodríguez-Yunta, Luis (2012). “¿Por qué nadie habla ya de las bases de datos bibliográficas 'clásicas'?". Anuario ThinkEPI, v. 6, pp. 130-134. http://recyt.fecyt.es/index.php/ThinkEPI/article/view/30403

Gimeno-Sieres, Elvira (2005). Repertorio y análisis documental de revistas españolas de farmacia y farmacología. Tesis doctoral. Valencia: Universitat de València.

http://hdl.handle.net/10550/15183

González-de-Dios, Javier; Flores-Canoura, Ángeles; Jiménez-Villa, Josep; Gutiérrez-Fuentes, José-Antonio (2011). "Qué revistas médicas españolas leen y cómo se informan los médicos de atención primaria". Atención primaria, v. 43, n. 12 , pp. 629-636.

http://www.elsevier.es/es-revista-atencion-primaria-27articulo-que-revistas-medicas-espanolas-leen-90055357 http://dx.doi.org/10.1016/j.aprim.2010.11.008

Jiménez-Contreras, Evaristo (2004). “La selección de la li- 
teratura científica en el ámbito biomédico: el factor de impacto". Educación médica, v. 7, n. Suplemento 2, pp. 27-35. http://hdl.handle.net/10760/12857

Llorente-Santacatalina, Sonia (2005). "Las revistas médicas españolas. Antecedentes y catálogo colectivo (1736-1850)". Documentación de las ciencias de la información, v. 28, pp. 211-256.

http://revistas.ucm.es/index.php/DCIN/article/view/ DCIN0505110211A

Melero, Remedios; Abad-García, María-Francisca (2008). "Revistas open access: características, modelos económicos y tendencias". BiD: textos universitaris de biblioteconomia $i$ documentació, 2008, n. 20.

http://bid.ub.edu/20meler2.htm

Melero, Remedios; Abad-García, María-Francisca; Abadal, Ernest; Amat, Carlos B.; Giménez-Martínez, Francesc; Rodríguez-Gairín, Josep-Manel; Rodríguez, Nerea (2009). "Dulcinea: iniciativa para el análisis de los derechos de copyright y autoarchivo de revistas científicas españolas". En: Fesabid 2009 XI Jornadas españolas de documentación, Zaragoza, 21 may 2009, pp. 117-121.

http://hdl.handle.net/10760/13160

Melero, Remedios; Hernández-San-Miguel, Javier (2014). "Acceso abierto a los datos de investigación, una vía hacia la colaboración científica". Revista española de documentación científica, 2014, v. 37, n. 4, pp. 1-11.

http://dx.doi.org/10.3989/redc.2014.4.1154

Osca, Julia; Civera, Cristina; Tortosa, Francisco; Quiñones, Elena; Peñaranda, María; López, Juan-José (2005). “Difusión de las revistas españolas de psicología en bases de datos nacionales e internacionales". Anales de documentación, n. 8, pp. 165-186.

http://revistas.um.es/analesdoc/article/view/1471/1531

Ponce-Aura, Concepción (2004). Análisis de la circulación de las revistas biomédicas españolas en bases de datos nacionales e internacionales. Tesis doctoral. Valencia: Universitat de València. http://roderic.uv.es/handle/10550/15218

Rozemblum, Cecilia; Unzurrunzaga, Carolina; Banzato, GuiIlermo; Pucacco, Cristian (2015). "Calidad editorial y calidad científica en los parámetros para inclusión de revistas científicas en bases de datos en acceso abierto y comerciales". Palabra clave (La Plata), v. 4, n. 2, pp. 64-80.

http://www.palabraclave.fahce.unlp.edu.ar/article/ download/PCv4n2a01/6597

Ruiz-Pérez, Rafael; Martín-Martín, Alberto; Delgado-López-Cózar, Emilio (2015). "Las revistas universitarias en el marco de los criterios de evaluación de la actividad investigadora en España". Revista española de documentación científica, v. 38, n. 2, p. e081.

http://dx.doi.org/10.3989/redc.2015.2.1191

Sancho, Rosa (2006). "Medición de las actividades de ciencia y tecnología. Estadísticas e indicadores empleados". Revista española de documentación científica, v. 24, n. 4, pp. 382-404.

http://dx.doi.org/10.3989/redc.2001.v24.i4.68

Sobrido-Prieto, María; Sobrido-Prieto, Natalia; GonzálezGuitián, Carlos; Pichel-Guerrero, María-Josefa; García-Sánchez, María-Matilde; Prieto-Díaz, Amelia (2005). "Revistas españolas de enfermería en bases de datos nacionales e internacionales". Index de enfermería, v. 14, n. 48-49, pp.74-77. http://goo.gl/DeDoHi

Vázquez-Valero, Manuela; Primo-Peña, Elena; Ronda-Laín, Concepción; García-Sicilia, José (2002). Las revistas científicas españolas de ciencias de la salud: directorio y análisis de la situación. Madrid: Consejo Superior de Investigaciones Científicas. ISBN: 8400080130

http://hdl.handle.net/10261/32524

Vázquez-Valero, Manuela; Urdín-Camino, Carmen; RománRomán, Adelaida (2003). "Las revistas españolas de ciencias de la salud frente a los criterios de calidad editorial Latindex". Revista española de documentación científica, v. 26, n. 4, pp. 418-432.

http://dx.doi.org/10.3989/redc.2003.v26.i4.143

\section{Anexo}

\begin{tabular}{|c|c|c|c|c|c|c|}
\hline \multicolumn{7}{|c|}{ Revistas que figuran en $\mathbf{2}$ bases de datos } \\
\hline Título & Ibecs & Medes & Medline & Embase & WoS & Scopus \\
\hline Ansiedad y estrés & $\mathrm{x}$ & & & & & $x$ \\
\hline Archivos de medicina & & & & $x$ & & $\mathrm{x}$ \\
\hline Archivos de medicina del deporte & $\mathrm{x}$ & & & & & $x$ \\
\hline Asclepio & $x$ & & & & & $\mathrm{X}$ \\
\hline Atención farmacéutica & & & & $x$ & & $x$ \\
\hline Avances en odontoestomatología & $x$ & & & & & $x$ \\
\hline Cancer and chemotherapy reviews & & & & $x$ & & $x$ \\
\hline Cirugía cardiovascular & & $x$ & & $x$ & & \\
\hline Cuadernos de medicina forense & $x$ & & & & & $x$ \\
\hline Cuadernos de psicología del deporte & $x$ & & & & & $\mathrm{x}$ \\
\hline Endodoncia & $x$ & & & & & $\mathrm{x}$ \\
\hline Farmacéutico hospitales, El & & & & $x$ & & $x$ \\
\hline Fisioterapia & $\mathrm{X}$ & & & & & $\mathrm{x}$ \\
\hline
\end{tabular}




\begin{tabular}{|c|c|c|c|c|c|c|}
\hline FMC Formación médica continuada en atención primaria & & & & $\mathrm{x}$ & & $x$ \\
\hline Imagen diagnóstica & & & & $x$ & & $x$ \\
\hline International journal of clinical and health psychology & & & & & $x$ & $x$ \\
\hline International journal of psychology and psychological therapy & $x$ & & & & & $\mathrm{x}$ \\
\hline Kranion & & & & $\mathrm{x}$ & & $\mathrm{x}$ \\
\hline Medicine & & & & $x$ & & $\mathrm{x}$ \\
\hline Metas de enfermería & $\mathrm{x}$ & $x$ & & & & \\
\hline Papeles del psicólogo & $\mathrm{x}$ & & & & & $\mathrm{x}$ \\
\hline Pediatría integral & & & & $\mathrm{x}$ & & $\mathrm{x}$ \\
\hline Revisiones en cáncer & & & & $\mathrm{x}$ & & $\mathrm{x}$ \\
\hline Revista andaluza de medicina del deporte & $\mathrm{x}$ & & & & & $\mathrm{x}$ \\
\hline Revista científica de la Sociedad Española de Enfermería Neurológica & & & & $x$ & & $\mathrm{x}$ \\
\hline Revista de la Asociación Española de Especialistas en Medicina del Trabajo & $\mathrm{x}$ & & & & & $\mathrm{x}$ \\
\hline Revista de la Asociación Española de Neuropsiquiatría & $\mathrm{x}$ & $\mathrm{x}$ & & & & \\
\hline Revista de logopedia, foniatría y audiología & $\mathrm{x}$ & & & & & $\mathrm{x}$ \\
\hline Revista de osteoporosis y metabolismo mineral & & $x$ & & $x$ & & \\
\hline Revista de patología respiratoria & $\mathrm{x}$ & & & $\mathrm{x}$ & & \\
\hline Revista de psicodidáctica & & & & & $\mathrm{X}$ & $\mathrm{X}$ \\
\hline Revista española de cirugía oral y maxilofacial & $\mathrm{x}$ & & & & & $\mathrm{x}$ \\
\hline Revista española de drogodependencias & $x$ & & & & & $\mathrm{x}$ \\
\hline Revista española de medicina legal & $\mathrm{x}$ & & & & & $\mathrm{x}$ \\
\hline Revista española de nutrición humana y dietética & & & & $\mathrm{x}$ & & $x$ \\
\hline Revista internacional de medicina y ciencias de la actividad fisica y del deporte & & & & & $\mathrm{x}$ & $\mathrm{x}$ \\
\hline Seminarios de la Fundación Española de Reumatología & & & & $\mathrm{X}$ & & $\mathrm{x}$ \\
\hline Todo hospital & $\mathrm{x}$ & & & & & $x$ \\
\hline Translational biomedicine & & & & $\mathrm{x}$ & & $\mathrm{x}$ \\
\hline \multicolumn{7}{|l|}{ Revistas que figuran en 3 bases de datos } \\
\hline Título & lbecs & Medes & Medline & Embase & WoS & Scopus \\
\hline Actualizaciones en anestesiología y reanimación & $\mathrm{x}$ & & & $x$ & & $\mathrm{x}$ \\
\hline Anales de psicología & $\mathrm{x}$ & & & & $\mathrm{x}$ & $\mathrm{x}$ \\
\hline Ars pharmaceutica & $\mathrm{x}$ & & & $\mathrm{x}$ & & $\mathrm{x}$ \\
\hline Cirugía pediátrica & $x$ & & $x$ & $x$ & & \\
\hline Cirugía plástica ibero-latinoamericana & $x$ & $x$ & & & & $x$ \\
\hline Diagnóstico prenatal & $\mathrm{x}$ & & & $\mathrm{x}$ & & $\mathrm{x}$ \\
\hline Diálisis y trasplante & & $x$ & & $\mathrm{x}$ & & $x$ \\
\hline Dolor, investigación, clínica \& terapéutica & & $x$ & & $x$ & & $\mathrm{x}$ \\
\hline Drugs of the future & & & & $x$ & $x$ & $\mathrm{x}$ \\
\hline Fundación Educación Médica & $\mathrm{x}$ & $x$ & & & & $x$ \\
\hline Enfermería global & $\mathrm{x}$ & $x$ & & & & $x$ \\
\hline European journal of anatomy, The & $\mathrm{x}$ & & & $x$ & & $x$ \\
\hline European journal of psychology applied to legal context & $x$ & & & & $x$ & $x$ \\
\hline Index de enfermería & $\mathrm{x}$ & $x$ & & & & $x$ \\
\hline Journal of optometry & & & $x$ & $x$ & & $x$ \\
\hline Medicina cutánea iberolatinoamericana & $x$ & & & $x$ & & $x$ \\
\hline Medicina preventiva & $x$ & $x$ & & & & $x$ \\
\hline Nutrición clínica y dietética hospitalaria & $x$ & $x$ & & & & $x$ \\
\hline Pediatria catalana & $x$ & & & $\mathrm{x}$ & & $x$ \\
\hline Pediatría de atención primaria & $x$ & $x$ & & & & $x$ \\
\hline Pharmaceutical care España & $x$ & & & $x$ & & $x$ \\
\hline Pharmacy practice & $x$ & & & $x$ & & $x$ \\
\hline Psicología conductual & $x$ & & & & $x$ & $x$ \\
\hline Psicológica & $x$ & & & & $x$ & $x$ \\
\hline
\end{tabular}




\begin{tabular}{|c|c|c|c|c|c|c|}
\hline Psicooncología & $x$ & & & $\mathrm{x}$ & & $x$ \\
\hline Psiquiatría biológica & $x$ & $\mathrm{x}$ & & $x$ & & \\
\hline Rehabilitación & $x$ & $x$ & & & & $x$ \\
\hline Revista de fitoterapia & $x$ & & & $x$ & & $x$ \\
\hline Revista de psicología del deporte & $\mathrm{x}$ & & & & $x$ & $x$ \\
\hline Revista de toxicología & $x$ & & & $\mathrm{x}$ & & $\mathrm{x}$ \\
\hline Revista del laboratorio clínico & $\mathrm{x}$ & & & $x$ & & $x$ \\
\hline Revista española de patología & $x$ & $\mathrm{x}$ & & $x$ & & \\
\hline SD. Revista médica internacional sobre el síndrome de Down & $x$ & & & $x$ & & $\mathrm{x}$ \\
\hline Spanish journal of psychology, The & $\mathrm{x}$ & & & & $x$ & $x$ \\
\hline Trauma & $\mathrm{x}$ & & & $x$ & & $x$ \\
\hline Vacunas. Investigación y práctica & & $x$ & & $x$ & & $x$ \\
\hline \multicolumn{7}{|l|}{ Revistas que figuran en 4 bases de datos } \\
\hline Título & Ibecs & Medes & Medline & Embase & Wos & Scopus \\
\hline Acta pediátrica española & $x$ & $x$ & & $x$ & & $x$ \\
\hline AIDS reviews & & & $x$ & $x$ & $x$ & $x$ \\
\hline Anales de la Real Academia Nacional de Farmacia & $x$ & $\mathrm{x}$ & & $x$ & & $x$ \\
\hline Angiología & $x$ & $x$ & & $x$ & & $x$ \\
\hline Apunts. Medicina de l'esport & $x$ & $x$ & & $x$ & & $x$ \\
\hline Archivos de prevención de riesgos laborales & $\mathrm{x}$ & & $x$ & $x$ & & $x$ \\
\hline Avances en diabetología & $x$ & $x$ & & $\mathrm{x}$ & & $x$ \\
\hline Clínica e investigación en ginecología y obstetricia & $x$ & $x$ & & $x$ & & $x$ \\
\hline Drugs of today & & & $x$ & $x$ & $x$ & $x$ \\
\hline Emergencias & $x$ & $x$ & & & $x$ & $x$ \\
\hline Enfermería intensiva & $x$ & & $x$ & $\mathrm{x}$ & & $x$ \\
\hline European journal of psychiatry, The & $x$ & & & $x$ & $x$ & $x$ \\
\hline Gerokomos & $x$ & $x$ & & $x$ & & $x$ \\
\hline Hipertensión y riesgo vascular & $x$ & $x$ & & $x$ & & $x$ \\
\hline Histology and histopathology & & & $x$ & $x$ & $x$ & $x$ \\
\hline Inmunología & $x$ & $x$ & & $x$ & & $x$ \\
\hline International journal of developmental biology, The & & & $\mathrm{x}$ & $\mathrm{x}$ & $\mathrm{x}$ & $\mathrm{x}$ \\
\hline Journal of investigational allergology \& clinical immunology & $x$ & & $x$ & $x$ & $x$ & \\
\hline Matronas profesión & $\mathrm{x}$ & $\mathrm{x}$ & & $x$ & & $x$ \\
\hline Medicina e historia & $\mathrm{x}$ & & $\mathrm{x}$ & $x$ & & $x$ \\
\hline Progresos de obstetricia y ginecología & $x$ & $x$ & & $x$ & & $x$ \\
\hline Revista de calidad asistencial & $x$ & $\mathrm{x}$ & $x$ & $x$ & & \\
\hline Revista de derecho y genoma humano & $x$ & & $x$ & $x$ & & $x$ \\
\hline Revista de la Sociedad Española del Dolor & $\mathrm{x}$ & $x$ & & $\mathrm{x}$ & & $\mathrm{x}$ \\
\hline Revista española de pediatría & $x$ & $x$ & & $\mathrm{x}$ & & $\mathrm{x}$ \\
\hline Revista iberoamericana de fertilidad y reproducción humana & $\mathrm{x}$ & $\mathrm{x}$ & & $\mathrm{x}$ & & $x$ \\
\hline Revista internacional de andrología & $x$ & $\mathrm{x}$ & & & $x$ & $x$ \\
\hline \multicolumn{7}{|l|}{ Revistas que figuran en 5 bases de datos } \\
\hline Título & Ibecs & Medes & Medline & Embase & Wos & Scopus \\
\hline Acta otorrinolaringológica española & $x$ & $\mathrm{x}$ & $\mathrm{x}$ & $\mathrm{x}$ & & $\mathrm{x}$ \\
\hline Actas dermo-sifiliográficas & $x$ & $\mathrm{x}$ & $x$ & $x$ & & $x$ \\
\hline Allergologia et immunopathologia & $\mathrm{x}$ & & $x$ & $x$ & $x$ & $x$ \\
\hline Archivos de la Sociedad Española de Oftalmología & $x$ & $x$ & $x$ & $x$ & & $x$ \\
\hline Archivos españoles de urología & $x$ & $x$ & $x$ & $x$ & & $x$ \\
\hline Clínica e investigación en arteriosclerosis & $x$ & $x$ & $x$ & $x$ & & $x$ \\
\hline Clinical and translational oncology & $x$ & & $x$ & $x$ & $x$ & $x$ \\
\hline Cuadernos de bioética & $x$ & $x$ & $\mathrm{x}$ & $x$ & & $\mathrm{x}$ \\
\hline
\end{tabular}




\begin{tabular}{|c|c|c|c|c|c|c|}
\hline Dynamis & $x$ & & $x$ & $x$ & $x$ & $x$ \\
\hline Endocrinología y nutrición & $x$ & $x$ & $x$ & $x$ & & $x$ \\
\hline Enfermería clínica & $x$ & $x$ & $x$ & $x$ & & $x$ \\
\hline Farmacia hospitalaria & $x$ & $x$ & $x$ & $x$ & & $x$ \\
\hline International microbiology & $x$ & & $x$ & $x$ & $x$ & $x$ \\
\hline Journal of physiology and biochemistry & $x$ & & $x$ & $x$ & $x$ & $x$ \\
\hline Medicina oral, patología oral y cirugía bucal & $x$ & & $x$ & $x$ & $x$ & $x$ \\
\hline Medicina paliativa & $x$ & $x$ & & $x$ & $x$ & $x$ \\
\hline Psicothema & $x$ & & $x$ & $x$ & $x$ & $x$ \\
\hline Radiología & $x$ & $x$ & $x$ & $x$ & & $x$ \\
\hline Reumatología clínica & $x$ & $x$ & $x$ & $x$ & & $x$ \\
\hline Revista de psiquiatría y salud mental & $x$ & $x$ & $x$ & $x$ & $x$ & \\
\hline Revista española de anestesiología y reanimación & $x$ & $x$ & $x$ & $x$ & & $x$ \\
\hline Revista española de cirugía ortopédica y traumatología & $x$ & $x$ & $x$ & $x$ & & $x$ \\
\hline Revista española de geriatría y gerontología & $x$ & $x$ & $x$ & $x$ & & $x$ \\
\hline Revista española de sanidad penitenciaria & $x$ & $\mathrm{x}$ & $x$ & $x$ & & $x$ \\
\hline Revista iberoamericana de micología & $x$ & & $x$ & $x$ & $x$ & $x$ \\
\hline Revista ROL de enfermería & $x$ & $x$ & $x$ & $x$ & & $x$ \\
\hline Semergen & $x$ & $x$ & $x$ & $x$ & & $x$ \\
\hline \multicolumn{7}{|l|}{ Revistas que figuran en 6 bases de datos } \\
\hline Título & Ibecs & Medes & Medline & Embase & Wos & Scopus \\
\hline Actas españolas de psiquiatría & $x$ & $x$ & $\mathrm{x}$ & $x$ & $x$ & $x$ \\
\hline Actas urológicas españolas & $x$ & $x$ & $x$ & $x$ & $x$ & $x$ \\
\hline Adicciones & $x$ & $x$ & $x$ & $x$ & $x$ & $x$ \\
\hline Anales de pediatría & $x$ & $x$ & $x$ & $x$ & $x$ & $x$ \\
\hline Anales del Sistema Sanitario de Navarra & $x$ & $x$ & $x$ & $x$ & $x$ & $x$ \\
\hline Archivos de bronconeumología & $x$ & $x$ & $x$ & $x$ & $x$ & $x$ \\
\hline Atención primaria & $x$ & $x$ & $x$ & $x$ & $x$ & $x$ \\
\hline Cirugía española & $x$ & $x$ & $x$ & $x$ & $x$ & $x$ \\
\hline Enfermedades infecciosas y microbiología clínica & $\mathrm{x}$ & $\mathrm{x}$ & $\mathrm{x}$ & $\mathrm{x}$ & $\mathrm{x}$ & $x$ \\
\hline Gaceta sanitaria & $x$ & $x$ & $x$ & $x$ & $x$ & $x$ \\
\hline Gastroenterología y hepatología & $x$ & $x$ & $x$ & $x$ & $x$ & $x$ \\
\hline Medicina clínica & $x$ & $x$ & $x$ & $x$ & $x$ & $x$ \\
\hline Medicina intensiva & $x$ & $x$ & $x$ & $x$ & $x$ & $x$ \\
\hline Nefrología & $x$ & $x$ & $x$ & $x$ & $x$ & $x$ \\
\hline Neurocirugía & $x$ & $x$ & $x$ & $x$ & $x$ & $x$ \\
\hline Neurología & $x$ & $x$ & $x$ & $x$ & $x$ & $x$ \\
\hline Nutrición hospitalaria & $x$ & $x$ & $x$ & $x$ & $x$ & $x$ \\
\hline Revista clínica española & $x$ & $x$ & $x$ & $x$ & $x$ & $x$ \\
\hline Revista de neurología & $x$ & $x$ & $x$ & $x$ & $x$ & $x$ \\
\hline Revista española de cardiología & $x$ & $x$ & $x$ & $x$ & $x$ & $x$ \\
\hline Revista española de enfermedades digestivas & $x$ & $\mathrm{x}$ & $\mathrm{x}$ & $x$ & $\mathrm{x}$ & $x$ \\
\hline Revista española de medicina nuclear e imagen molecular & $x$ & $\mathrm{x}$ & $\mathrm{x}$ & $\mathrm{x}$ & $\mathrm{x}$ & $\mathrm{x}$ \\
\hline Revista española de quimioterapia & $x$ & $x$ & $x$ & $x$ & $x$ & $x$ \\
\hline Revista española de salud pública & $x$ & $x$ & $x$ & $x$ & $x$ & $x$ \\
\hline
\end{tabular}

